\title{
Health-related quality of life among Swedish children with Juvenile Idiopathic Arthritis: parent-child discrepancies, gender differences and comparison with a European cohort
}

\author{
Veronica Lundberg ${ }^{*}$ (1) and Catharina Eriksson
}

\begin{abstract}
Background: This study investigates gender differences in self-reports and between parent and child reports in Healthrelated Quality of Life (HRQOL), measured with disease-specific and generic instruments for chronic disease. Comparison of HRQOL results in this Juvenile Idiopathic Arthritis (JIA) sample to a European cohort of children with JIA and one of children with other health conditions are also made.

Methods: Fifty-three children with juvenile idiopathic arthritis (JIA), aged 8-18 years, and their parents completed the condition-specific DISABKIDS for JIA, and the DISABKIDS generic instrument for chronic conditions (DCGM-37) in a cross-sectional study. European reference data were used for comparison of child and parental reports.

Results: Child self-reports in DCGM-37 and DISABKIDS for JIA showed no gender differences. Parental and child reports of the child's HRQOL differed only in DCGM-37; this was among girls who scored their independence $(p=0.03)$, physical limitation $(p=0.01)$, social exclusion $(p=0.03)$, emotions $(p<0.01)$, and general transformed score $(p<0.01)$ higher than did their parents. Our sample of children with JIA reported more physical limitation compared to samples of European children with JIA $(p=0.01)$, European children with chronic conditions $(p<0.01)$, and their parents $(p=0$. 01 and $p<0.01$ ). The Swedish children reported more problem with understanding compared to the European JIA sample $(p=0.03)$. Swedish parents perceived their children's independence significantly lower than did the European parents of JIA children $(p<0.01)$, as well as European parents of children with chronic conditions $(p=0.03)$. The Swedish parents also perceived their children to have significantly lower social inclusion $(p<0.05)$ and general transformed score $(p=0.04)$, in comparison to European parents of children with chronic conditions. Conclusions: Parent-child differences in assessment of quality of life depend on the HRQOL instrument used, especially among girls. In comparison to European cohorts, our sample of children with JIA experienced more physical limitations and less understanding.
\end{abstract}

Keywords: Gender, Informant discrepancies, Paediatrics, Questionnaire

* Correspondence: veronica.lundberg@vll.se

Department of Clinical Sciences, Pediatrics, Umeå University, SE 90185 Umeå, Sweden 


\section{Background}

Juvenile idiopathic arthritis (JIA) encompasses all forms of arthritis that begin before the age of 16 years, persists for more than 6 weeks, and are of unknown cause [1-3]. JIA is classified into seven disease subtypes by to the International League of Associations for Rheumatology (ILAR) [4]. Improvement in functional outcomes has been documented, and is due to progress in disease management [1]. Still, growing up with JIA can be associated with risk for numerous functional limitations, e.g., swollen joints, medication side effects, pain, fatigue $[3,5,6]$, and visual impairment $[3,6]$. Children with JIA are more likely to report a lower health related quality of life (HRQOL), compared to normative data [2, 3, 7].

HRQOL measures can be divided into conditionspecific measures, generic, or generic instruments for chronic conditions. Condition-specific instruments address aspects related to a specific condition, its treatment, or symptoms. Generic instruments are suitable to measure HRQOL across both healthy populations and populations with medical health conditions, while generic instruments for chronic conditions assesses HRQOL only among children with various chronic health conditions. In our previous study, we used the generic HRQOL instrument Pediatric Quality of Life inventory (PedsQL) in the same sample as in this present article, and found that more than half of girls and boys with JIA experienced suboptimal HRQOL compared to a healthy population [7].

Differences between self-report and parent-report (proxy-rater) has been demonstrated in HRQOL measurements of children with and without health conditions $[8,9]$. However, there are mixed findings in earlier studies that consider gender differences in parental versus child reports. They suggest that a child's gender may not be related to informant discrepancies, except in specific populations [10]. Our previous study showed gender differences between parent and child self-reports for the child HRQOL in JIA children, measured with the generic PedsQL. The most significant differences were among girls, who rated their HRQOL better in most subdomains compared to their parents [7].

To our knowledge, there have been no studies that evaluate parent-child discrepancies among different types of HRQOL instruments, e.g., disease-specific and generic HRQOL instruments for chronic conditions.

\section{Objectives}

The primary aims of the present study were to evaluate child self-reported and parent-reported HRQOL with instruments for chronic conditions, and a conditionspecific HRQOL instrument for JIA. We specifically wanted to evaluate for gender differences in HRQOL self-report, and to explore gender differences in parent report of a child's HRQOL. The secondary aims were to compare the HRQOL results in this JIA sample to a European cohort of children with JIA and one of children with other health conditions.

\section{Methods \\ Study design and study population}

This cross-sectional questionnaire survey included children and adolescents registered with a diagnosis of JIA at the Department of Child and Adolescent Medicine, Norrlands University Hospital, Umeå, Sweden. The study population included all eligible children, identified through clinical appointment schedules at routine outpatient appointments, between September 2009 and December 2010.

\section{Participants}

Eligibility criteria were children aged 8 to 18 years with a JIA diagnosis defined by the ILAR classification criteria [4]. Of 69 eligible children, 56 children and their parents agreed to participate. The reason for declining participation was unknown in six families, four parents said that their child only had small problems due to JIA, two cited a lack of time, and one child was discharged from the clinic. Three were excluded because of a missing parental report. Therefore, 53 children (38 girls and 15 boys) remained as the study group. Of the participating parents, 37 were mothers, 12 were fathers, and in four families the mothers and fathers completed the questionnaires together. For nine of the girls, at least one additional diagnosis was reported by the child or parent, i.e., uveitis, overweight, attention-deficit/hyperactivity disorder, asthma, dermatitis, Turners syndrome, celiac disease, Crohn's disease, or thyroid gland disease. Among five of the boys, at least one additional diagnosis was reported, i.e., asthma, allergy, panic disorder, Raynaud's syndrome, or lactose intolerance.

\section{Measures \\ HRQOL}

The DISABKIDS generic instrument for chronic conditions (DCGM-37) consists of 37 items assigned to six subscales: independence, emotional, social inclusion, social exclusion, physical limitation, and medication. DCGM-37 was tested in 2002-2003 cross-culturally in seven European countries, including Sweden. The findings with respect to internal consistency (Chronbachs'Alpha: 0.700.87 in child version and $0.77-0.90$ in proxy version) and test-retest reliability $(0.71-0.83)$ has been demonstrated. The results indicate that DCGM-37 is suitable for HRQOL comparisons in children and adolescents with different diseases and across cultures [11-13]. The condition-specific DISABKIDS for JIA addresses arthritisspecific symptoms and limitations. Its 12 items are assigned to two sub-scales: impact, and understanding. The internal consistency of each sub-scale was between 0.73 and 0.87 , in a field study among children with JIA 
[13]. Both the JIA DISABKIDS and DCGM-37 are suitable for use between ages 8 and 16 years. Each item relates to symptoms during the last four weeks, and was answered on a five-point Likert scale between 1 (never) to 5 (always). In our sample, when a child or parent registered an odd number, the score was rounded to the inferior option. The scale scores were transformed with the syntaxes for DISABKIDS scales, to a range of 0 to 100, with higher values indicating better HRQOL [12]. Both self-report and an equivalent parent version was included.

European reference data, obtained from the DISABKIDS manual [12] were used for comparison with the Swedish sample of JIA patients. The reference data, based on a field study, included a European cohort of $n=148$ children with JIA, and a cohort of $n=1152$ participants with the following chronic conditions; arthritis, asthma, atopic dermatitis, cerebral palsy, cystic fibrosis, diabetes and epilepsy [12].

Disability was measured by the Childhood Health Assessment Questionnaire (CHAQ) [14], composed of 30 items that measure eight functional areas. Each question was scored on a four-point scale between 0 (no difficulty) and 3 (unable to do). The question with the highest score determined the score for that functional area. The scores for each of the eight functional areas were averaged to calculate a Disability Index (DI) that ranged from 0 (no disability) to 3 (disabled) [14]. Suggested cutoff levels for disability are at medians of 0.13 (mild), 0.63 (mild-to-moderate), and 1.75 (moderate) [15]. The original CHAQ showed internal reliability (Chronbachs'Alpha 0.94) and test-retest reliability with Spearman's correlation coefficient of 0.8 and convergent validity between Kendall's tau; 0.54-.77. The Swedish version of CHAQ showed internal reliability of Cronbach's alpha 0.89 , test-retest reliability for the disability index was high $(\mathrm{r}(\mathrm{S})=0.92, \mathrm{r}(\mathrm{P})=0.97 ; p<0.00001)[14,16]$.

Disease duration in years was calculated from medical record data, based on the date of diagnosis and according to ILAR classification criteria.

Disease activity was measured by a paediatric rheumatologist who assessed global disease activity into categories of no, mild, moderate, or high. Mild disease activity was defined as between no activity and moderate activity, moderate activity meant the child needed changes in medical therapy, and high activity captured severe immunologic and clinical activity. Also erythrocyte sedimentation rate (ESR) in $\mathrm{mm} /$ hour was used.

Medications were compiled into four categories: 1) disease modifying anti-rheumatic drugs (DMARDs), e.g., azathioprine, cyclosporine, hydroxychloroquine, methotrexate, and sulfasalazine; 2) non-steroidal anti-inflammatory drugs (NSAIDs); 3) biological medications, e.g., adalimumab, anakinra, etanercept, infliximab and rituximab; and 4) corticosteroid therapy (Table 1).

\section{Procedures}

Families who agreed to participate in the study completed a mental health questionnaire; the Strengths and Difficulties Questionnaire and three HRQOL questionnaires, of which the DISABKIDS for JIA and the DCGM-37 were included in this study. The third HRQOL questionnaire, PedsQL is presented in an earlier article [7]. All these questionnaires were answered prior to a scheduled medical visit at the paediatric outpatient clinic. Parents and children completed the questionnaires separately, and a research assistant was available for the child. The CHAQ is used routinely in clinical practice, and was answered by children and their parents at home before the outpatient clinic visit. Estimated time to complete all of the study questionnaires was 20-40 min for each child, plus another ten minutes for the CHAQ to be completed at home. Nor the children or parents were reimbursed for their time participating in this study.

\section{Statistics}

Data from girls, boys, and their parents were analysed separately and as one group. Results of the descriptive analysis are presented as median and range, or numbers (n) and percentages (\%). HRQOL results are presented as mean, standard deviation (SD), median (Md), and range. Mann Whitney $U$ test was used to analyse gender differences in characteristics and to compare the median of HRQOL between boys and girls. The Wilcoxon signed-rank test was used to analyse differences between self-reports and parental reports of HRQOL. Student's one sample $t$-test with hypothesized mean values obtained from the European reference data in tables 4-11, $4-12,4-15,4-16$ and $4-278$ in the DISABKIDS manual were used to evaluate for HRQOL differences between the Swedish JIA sample and European samples [12]. The analyses were performed using Statistical Package for the Social Sciences (SPSS) version 18, 19 and 22. Statistical significance was defined as $P<0.05$.

\section{Results \\ Sample characteristics}

The 53 children with JIA had a median age of 14 years (range 8-18). The paediatric rheumatologist categorized no or low disease activity among $87 \%$ of the girls and $86 \%$ of the boys. Girls and boys differed significantly only in JIA sub-diagnosis (Table 1).

\section{Generic HRQOL for chronic disease}

There were gender differences between child self-report and parental report in DCGM-37. The girls and their parents differed significantly in the subscales of independence, physical limitation, social exclusion, emotion, and general transformed score. Among the boys, there were no 
Table 1 Characteristics of 53 children with juvenile idiopathic arthritis aged 8-18 years

\begin{tabular}{|c|c|c|c|}
\hline & Girls $(n=38)$ & Boys $(n=15)$ & $P$-value ${ }^{a}$ \\
\hline Age in years, Md (range) & $13(8-18)$ & $15(10-18)$ & 0.51 \\
\hline $8-12$ years, $n(\%)$ & $16(42)$ & $5(33)$ & \\
\hline $13-18$ years, $n(\%)$ & $22(58)$ & $10(67)$ & \\
\hline CHAQ Disability Index ${ }^{\mathrm{b}}, \mathrm{Md}$ (range) & $0.50(0-2.75)$ & $0.38(0-1.38)$ & 0.65 \\
\hline Categories, $n(\%)$ & & & 0.56 \\
\hline None-mild & $12(32)$ & $5(33)$ & \\
\hline Mild-moderate & $9(24)$ & $5(33)$ & \\
\hline Moderate & $13(34)$ & $5(33)$ & \\
\hline Severe & $3(8)$ & & \\
\hline Missing & $1(3)$ & & \\
\hline Pain VAS ${ }^{\complement}, 0-100$ Md (range) & $32(0-93)$ & $50(0-99)$ & 0.50 \\
\hline Well-being $\mathbf{V A S}^{\mathrm{d}}, 0-100 \mathrm{Md}$ (range) & $22(0-99)$ & $27(0-100)$ & 0.75 \\
\hline ILAR diagnosis ${ }^{\mathrm{e}}, \boldsymbol{n}(\%)$ & & & 0.01 \\
\hline Systemic arthritis & 1 (3) & $1(7)$ & \\
\hline Oligoarthritis & $16(42)$ & $3(20)$ & \\
\hline Polyarticular arthritis, $\mathrm{RF}^{\mathrm{f}}$-negative & $2(5)$ & & \\
\hline Polyarticular arthritis, $\mathrm{RF}^{f}$-positive & $8(21)$ & $1(7)$ & \\
\hline Psoriatic arthritis & $6(16)$ & & \\
\hline Enthesitis-related arthritis & $3(8)$ & $5(33)$ & \\
\hline Unspecified arthritis & $2(5)$ & $5(33)$ & \\
\hline \multicolumn{4}{|l|}{ Physical education participation, } \\
\hline$n(\%)$ & & & 0.08 \\
\hline Always & $12(32)$ & $9(60)$ & \\
\hline Sometimes & $16(42)$ & $4(27)$ & \\
\hline Not at all & $6(16)$ & $2(13)$ & \\
\hline Not relevant & $3(8)$ & & \\
\hline Missing & 1 (3) & & \\
\hline Disease duration in years Md (range) & $4.5(0-16)$ & $3.0(0-13)$ & 0.09 \\
\hline ESR $^{9}, \mathrm{~mm} /$ hour, Md (range) & $5(2-31)$ & $4(2-35)$ & 0.14 \\
\hline Disease activity categories, $n(\%)$ & & & 0.37 \\
\hline No & $13(34)$ & $8(53)$ & \\
\hline Low & $20(53)$ & $5(33)$ & \\
\hline Moderate & $4(10)$ & $2(13)$ & \\
\hline High & 0 & 0 & \\
\hline Missing & $1(3)$ & 0 & \\
\hline \multicolumn{4}{|l|}{ Medication at study visit ${ }^{h}, n(\%)$} \\
\hline DMARD & $24(63)$ & $7(47)$ & 0.70 \\
\hline NSAID & $25(66)$ & $8(53)$ & 0.42 \\
\hline Biologic medication & $4(10)$ & 0 & \\
\hline Corticosteroid therapy & $7(18)$ & 0 & \\
\hline Eye drops/corticosteroid & $2(5)$ & 0 & \\
\hline
\end{tabular}

\section{aann Whitney $U$ test}

${ }^{\mathrm{b}}$ Childhood Health Assessment Questionnaire Disability Index

'Visual Analogue Scale for pain during the past week; $0=$ no pain and $100=$ very severe pain

${ }^{d}$ Visual Analogue Scale for assessment of overall well-being; $0=$ very good and $100=$ very bad

e JIA- diagnosis defined by the International League of Associations for Rheumatology (ILAR) classification criteria

fRheumatoid factor

${ }^{9}$ Erythrocyte sedimentation rate

hDisease modifying anti-rheumatic drug (DMARD), Non-steroid anti-inflammatory drug (NSAID) 
differences between self-reports and parental reports on any subscales or for the total scale score. There were no differences between girls' and boys' self-report for any of the six DCGM-37 subscales, total general transformed score, or the 37 specific questions included in the questionnaire. However, among the parents' answers in the DCGM-37 social exclusion dimension, parents of boys reported higher (better) child scores than did parents of girls $(P=0.05)$ (Table 2).

\section{JIA-specific HRQOL}

Children reported a mean condition-specific impact score of 60.30 (SD 22.21) and an understanding mean score of 60.58 (SD 24.15). The parental mean impact score was 57.98 (SD 21.34); the understanding mean score was 63.83 (SD 25.79). No significant differences between genders were found in the self-report for condition-specific DISABKIDS for JIA questionnaire, or between the children and their parents.

\section{Comparison with the European reference data cohort}

On the DCGM-37, the Swedish JIA children scored significantly lower on the physical limitation subscale than both the European JIA children $(P=0.01)$ or European children with chronic conditions $(P<0.01)$. The parents reported significantly lower scores on child physical limitation compared to European parents of JIA children $(P=0.01)$ or European parents of children with chronic conditions $(P<0.01)$. The Swedish parents perceived their children's independence to be significantly lower than the European parents of children with JIA $(P<$ $0.01)$, as well as the European parents of children with a chronic condition $(P=0.03)$. In comparison to the Swedish sample, European parents of children with chronic conditions perceived their children to have significantly better social inclusion $(P<0.05)$ and general transformed score $(P=0.04)$ (Table 3$)$.

In the JIA-specific HRQOL instrument, our Swedish JIA children rated the understanding scale significantly lower than the European JIA sample: 60.58 (SD 24.15) versus 67.75 (SD 26.73) $(P=0.03)$.

\section{Discussion}

We found no differences between parental and child reports with the condition-specific HRQOL instrument DISABKIDS for JIA. Nor did we find a gender difference in children's self-reports. However, there were gender differences between parental and child reporting in DCGM-37; girls reported significantly better scores on several HRQOL sub-scales compared with parental reports. This confirms our previous finding in this same sample when the generic instrument PedsQL was used [7]. In the generic questionnaire PedsQL, the parentchild differences were also evident among the girls, who tended to reported better physical health, psychosocial health, and total HRQOL compared to their parents' reports [7]. Also in accordance with our previous results [7], the boys tended to score lower on several HRQOL sub-scales, in both the conditions-specific DISABKIDS for JIA and the DCGM-37, compared to their parents. Such a scoring difference was not significant in the present study. Some authors found gender differences, with lower parent-child agreement in mental health between girls and their parents $[10,17]$, while others found inconsistent results [10]. Differences in agreement that depend on whether the mother or father is the proxyrater were not explored in this study. A review by Upton et al. found a possible interaction of age and gender in parent-child agreement on HRQOL ratings; this depended on the gender of the parental proxy-rater. Fathers and mothers can have different perspectives on child health and behaviour, and sons and daughters might also differ in their relationship with each parents [8]. Mothers' and fathers' ratings correlate moderately, and bigger differences are seen between father/child ratings than for mother/child ratings [9].

Eiser et al. describe in their review, that a parent's own well-being may affect the report of their child's HRQOL and symptoms. If the parents report higher levels of emotional distress, they also report more negative perceptions of their child's HRQOL [9]. However, Haverman et al. found that the parents of children with JIA self-reported their own HRQOL similar to parents of healthy children [18]. Specific HRQOL subdomains are suggested to affect parent-child agreement, with better agreement in observable behaviours such as physical functioning than in internal symptoms such as pain, emotional distress, or fatigue [9]. For example, in the PedsQL the majority of items involve observable items and are suggested to reflect good agreement [8]. However, Upton et al. found divergent findings for parentchild agreement on specific subscales, e.g., observable characteristics such as physical health and social and emotional functioning in healthy children and children with different health conditions [8].

Child self-report may be more reliable for information regarding minor, short-term effects of treatment, moment-to-moment HRQOL and disease-specific symptoms, whereas parents report might reflects overall symptoms occurring over time $[9,19]$. Children might also report more symptoms, but less impact of their perceived difficulties than do their parents [19]. In children with a diagnosed medical condition, parental involvement and understanding increases regarding their child's health issues and well-being [8, 9]. Even level of disease activity seems to affect parent-child agreement among children with polyarticular JIA. If the child had active disease, the parent-child agreement was higher than 


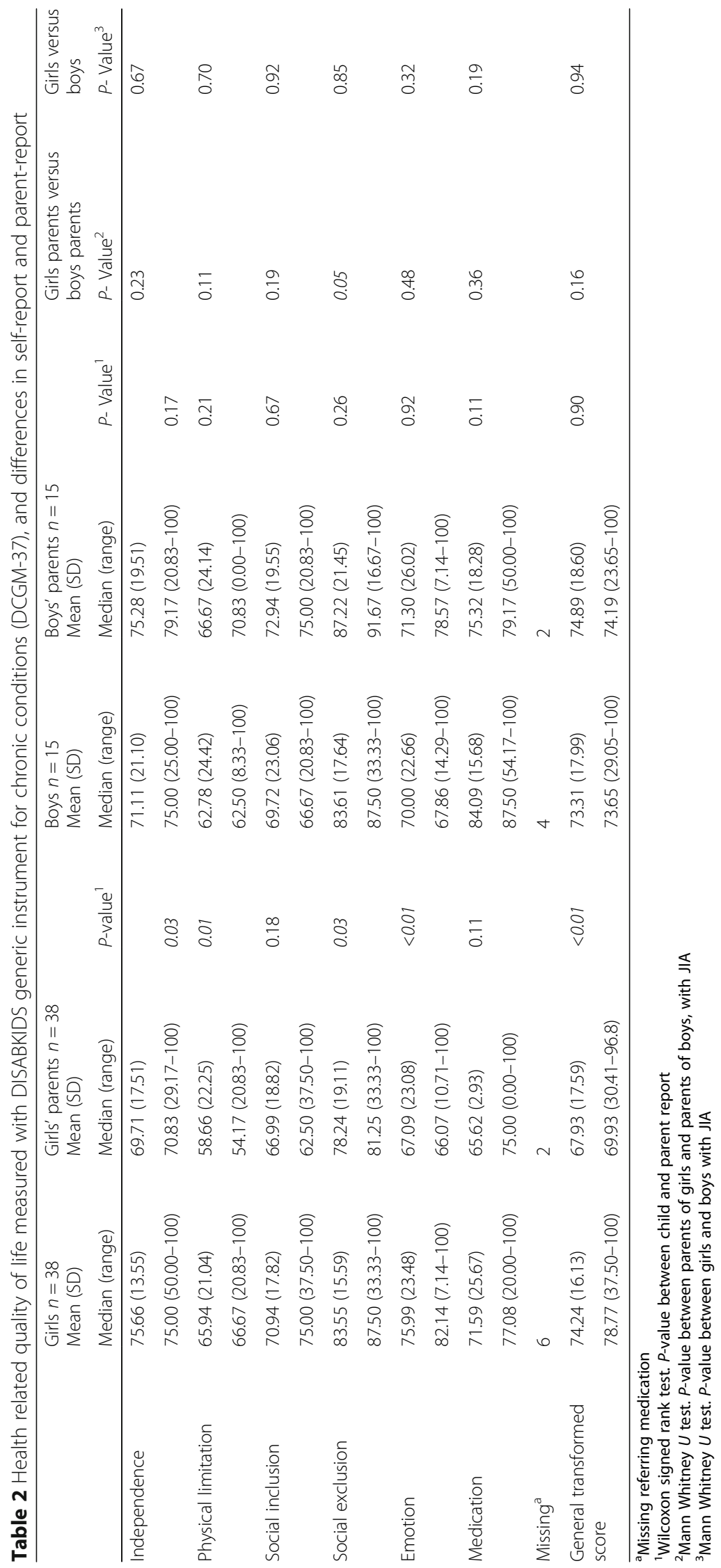




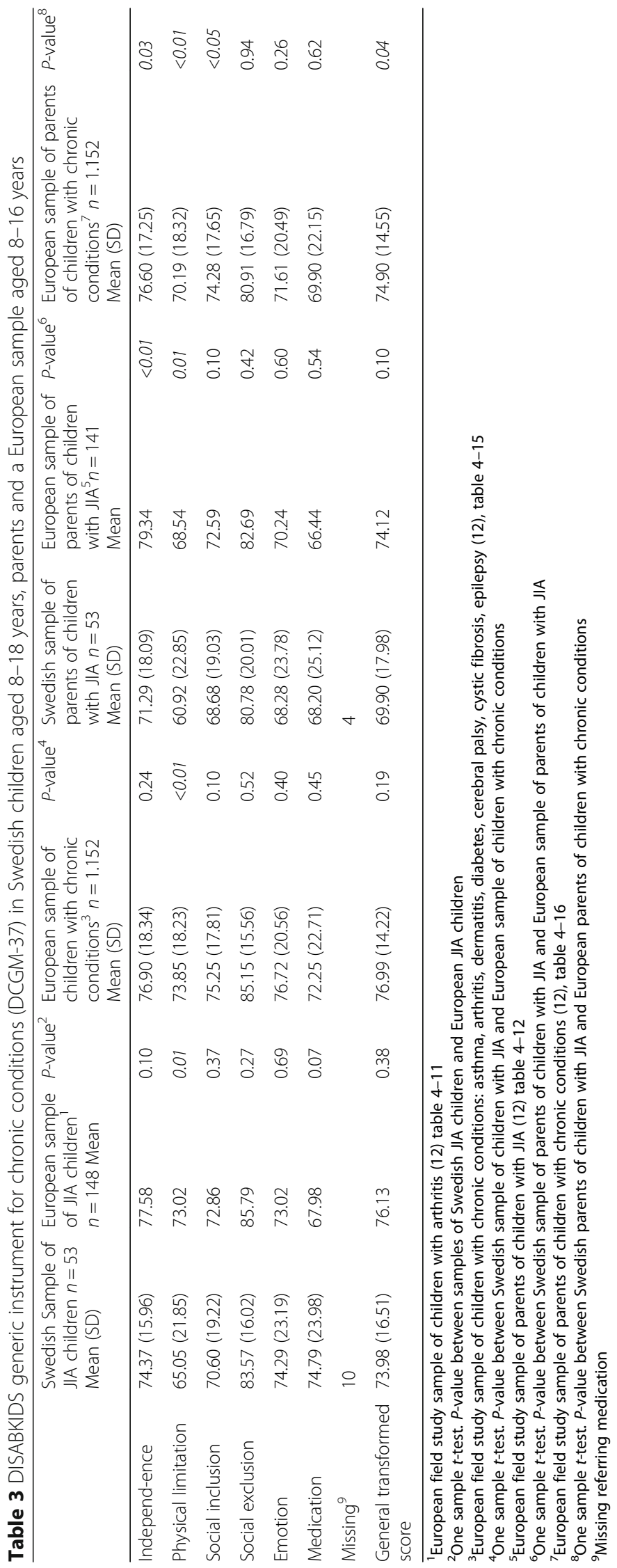


among children with inactive disease [20]. Parents of children with JIA might perceive their children as vulnerable, especially when the children have a shorter disease duration, higher degree of functional disability, or greater disease activity [18]. On the other hand, our sample of children did not differ in any of these characteristics. This view would therefore not explain the significant inter-rater differences on the DCGM-37 among girls. ILAR diagnosis was the only variable that differed among girls and boys in our sample. Parents are not in position to observe their children in environments outside the family, or how these influence the child's feelings and function on a daily basis. And this is especially true for school-aged children and adolescents [9]. Interrater agreement can depend on the developmental level and age of the child, as well as the characteristics of the illness [9]. Yet, the authors found divergent results in a review that compared parent-child agreement in relation to child's age. This ranged from no correlation with the child's age to lower concordance between parents and adolescent children [8]. Due to the small sample, we did not evaluate for age differences in our study.

The sub-scales in the condition-specific DISABKIDS for JIA are supposed to be sensitive to treatment and improvement of the condition. The subscales impact and understanding are related to the invisibility and fluctuation of JIA symptoms [12]. In comparison to the European sample of children with JIA, our Swedish sample rated their understanding subscale significantly lower. This implies greater feelings of social exclusion and lack of empathy by peers and teachers. This suggests that Swedish children with JIA need more interventions in health education within school-based services [12].

The Swedish children with JIA and their parents scored significantly lower on the child physical limitation DCGM37 subscale compared to the European children with JIA, European children with chronic conditions, and their parents. This indicates that our sample of children with JIA experience more functional limitations that are caused by JIA, and might need targeting intervention regarding exercise training [12]. Children with JIA have reduced anaerobic capacity and muscle strength. This results in physical, mental, and metabolic disorders. Exercise training is beneficial for JIA children, and leads to improved muscle strength, range of motion, quality of life, and reduced joint stiffness, without affecting disease activity [21]. Therefore, preventing physical limitations and supporting exercise training is essential in JIA. The Swedish parents perceive their children's independence to be significantly lower compared to European parents of children with JIA, and European parents of children with a chronic condition. Compared to Swedish parents, European parents of children with chronic conditions perceive their children to have better social inclusion and a higher general transformed score. Comparison between these samples should be interpreted with caution since the characteristics and eventual comorbidity of the European samples are not described in detail [12]. A selection bias might therefore be evident, since the participants in the Swedish sample might not be exchangeable to those in the European cohort. The clinical relevance of different HRQOL domains may also vary between subgroups within broader disease groups like JIA. This makes meaningful comparisons difficult [8]. We do not know what demands our sample requires in daily life, or if these requirements differ between European countries; e.g., physical demands in school physical education classes, sports or recreational activities.

Possible bias in the results could be present because DISABKIDS assesses children 8-16 years of age, and we included children 8-18 years of age. The reason for including even 16-18 year old participants for us, were to evaluate for differences in parent-child discrepancies between three different HRQOL instruments; this present study and our previous study with the same sample [7]. Also, in our sample there was a relatively high frequency of comorbidity related to JIA or unrelated conditions. These may have influenced our results. Four children declined participation because they only had minor symptoms of JIA. This might have biased our results towards a lower HRQOL in some subscales. This sample size was small, especially among the boys. Only 15 boys were available for the study, and the comparisons involving the boys might therefore be underpowered. The multiple comparisons might also have resulted in significant results by chance. Other possible confounders such as socioeconomic status or parental education were not measured in this study.

Extensive attention has thus been given to examining informant characteristics that affect parent-child differences. Our results in the present and an earlier study show that the HRQOL instrument used can also influence parent-child differences in HRQOL. Differences, especially among girls, were evident in generic HRQOL measurement in our previous study [7], and in the DISABKIDS generic HRQOL instrument for chronic conditions in this study. But no differences were evident in the conditions specific HRQOL instrument DISABKIDS for JIA. To our knowledge this is the first study investigating parent-child proxy-rate discrepancies in different HRQOL instruments. Inconsistent or no differences exist for many of the child and parent characteristics reviewed in earlier studies (e.g., child's age, gender, problem type, HRQOL subdomains, social desirability, child and parent health, relationship) with regard to parentchild differences in reports of the child's HRQOL. Knowledge of all available research is essential to take into account when evaluating child self-report of HRQOL. Integrating and analysing all such variables 
into paediatric healthcare might be impractical. However, implementation of routine HRQOL measurements in clinical care is an important goal for assessment of paediatric patients with chronic health conditions like JIA. This is especially the case since JIA involves fluctuating disease-activity, long-term treatment, common medication side effects, social and physical limitations, and comorbidities related or unrelated to JIA. Facilitating child self-report is essential when one considers the many variables that can affect parent-child differences in HRQOL measurement. Child self-report should be complemented by parent-report in order to give a more complete report of the child's health.

We agree with other authors $[8,9]$, that information from both the child and parent provides a richer picture and invaluable information. Therefore, both need to be included whenever evaluating a child's HRQOL. If the child is unable to self-report, a condition specific HRQOL measurement like DISABKIDS for JIA, might be preferable for proxy-report and best reflect the child's own opinion of their HRQOL at the time of evaluation. Routine HRQOL measurement in the clinic can promote targeted interventions from healthcare professionals. For example, interventions could be directed toward the physical limitations and understanding evident in our sample.

\section{Conclusions}

Differences in parent vs. child self-report were evident among girls with JIA when measured with the generic instrument for chronic disease, DCGM-37. These results may indicate that parent-child differences depend on the type of HRQOL instrument used. There was no gender differences among children's self-report on any of the HRQOL instruments used in this study. In comparison to a European cohort, Swedish children with JIA experience more physical limitation and problems with understanding.

\section{Acknowledgements}

The authors wish to thank all of the children and families who participated in the study and Viveca Lindh for supporting the initial work and data collection.

\section{Funding}

This work was financially supported by the Majblomman Foundation.

\section{Availability of data and materials}

The data that support the findings of this study are available from Viveca Lindh, PhD, Department of Nursing, Umeå University, Umeå, Sweden. But restrictions apply to the availability of these data, which were used under license for the current study, and so are not publicly available. Data are however available from the authors upon reasonable request and with permission of Viveca Lindh.

\section{Authors' contributions}

VLU participated in conception and design of the study, in acquisition of data, analysis and interpretation of data, and in drafting of the manuscript. CEl contributed in conception and design of the study, in the analysis and interpretation of data, and in editing the manuscript. Both authors provided critical revision of the manuscript, read and gave final approval of the manuscript to be published.

\section{Competing interests}

The authors declare they have no competing interests.

\section{Consent for publication}

Not applicable.

\section{Ethics approval and consent to participate}

Ethical approval was obtained from the regional ethical review board in Umeå, Sweden (Dnr 09-070 M). Informed consent was given by each family. The results were reported on group level, and did not reveal individual results. All questionnaires were numbered with a study ID, placed in a locked box after completion, and available only to the researchers responsible for the study.

\section{Publisher's Note}

Springer Nature remains neutral with regard to jurisdictional claims in published maps and institutional affiliations.

Received: 12 January 2017 Accepted: 28 March 2017

Published online: 12 April 2017

\section{References}

1. Ravelli A, Martini A. Juvenile idiopathic arthritis. Lancet. 2007;369(9563):767-78.

2. Prince $F H$, Otten MH, van Suijlekom-Smit LW. Diagnosis and management of juvenile idiopathic arthritis. BMJ. 2010;341:c6434.

3. Moorthy LN, Peterson MG, Hassett AL, Lehman TJ. Burden of childhoodonset arthritis. Pediatr Rheumatol Online J. 2010;8:20.

4. Petty RE, Southwood TR, Baum J, et al. Revision of the proposed classification criteria for juvenile idiopathic arthritis: Durban, 1997. J Rheumatol. 1998:25(10):1991-4.

5. Eyckmans $L$, Hilderson D, Westhovens $R$, et al. What does it mean to grow up with juvenile idiopathic arthritis? A qualitative study on the perspectives of patients. Clin Rheumatol. 2011;30(4):459-65.

6. Miller-Hoover S. Juvenile idiopathic arthritis: why do I have to hurt so much? J Infus Nurs. 2005:28(6):385-91.

7. Lundberg V, Lindh V, Eriksson C, et al. Health-related quality of life in girls and boys with juvenile idiopathic arthritis: self- and parental reports in a cross-sectional study. Pediatr Rheumatol Online J. 2012;10(1):33.

8. Upton P, Lawford J, Eiser C. Parent-child agreement across child healthrelated quality of life instruments: a review of the literature. Qual Life Res. 2008;17(6):895-913.

9. Eiser C, Varni JW. Health-related quality of life and symptom reporting: similarities and differences between children and their parents. Eur J Pediatr. 2013;172(10):1299-304.

10. De Los Reyes A, Kazdin AE. Informant discrepancies in the assessment of childhood psychopathology: a critical review, theoretical framework, and recommendations for further study. Psychol Bull. 2005;131(4):483-509.

11. Simeoni M, Schmidt S, Muehlan $\mathrm{H}$, et al. Field testing of a European quality of life instrument for children and adolescents with chronic conditions: the 37-item DISABKIDS Chronic Generic Module. Qual Life Res. 2007;16(5):88193.

12. Schmidt S, Peterson C, Muhlan $\mathrm{H}$, et al. The DISABKIDS QuestionnairesHandbook incl. CD-Rom. Lengerich: Pabst Science Publishers; 2006.

13. Baars R, Atherton C, Koopman H, et al. The European DISABKIDS project: development of seven condition-specific modules to measure health related quality of life in children and adolescents. Health Qual Life Outcomes. 2005;3:70.

14. Singh G, Athreya B, Fries J, Goldsmith D. Measurement of health status in children with juvenile rheumatoid arthritis. Arthritis Rheum. 1994;37(12): 1761-9.

15. Dempster H, Porepa M, Young N, Feldman BM. The clinical meaning of functional outcome scores in children with juvenile arthritis. Arthritis Rheum. 2001;44(8):1768-74.

16. Andersson Gäre B, Fasth A, Wiklund I. Measurement of functional status in juvenile chronic arthritis: evaluation of a Swedish version of the Childhood Health Assessment Questionnaire. Clin Exp Rheumatol. 1993;11(5):569-76.

17. Waters E, Stewart-Brown S, Fitzpatrick R. Agreement between adolescent self-report and parent reports of health and well-being: results of an epidemiological study. Child Care Health Dev. 2003;29(6):501-9.

18. Haverman $\mathrm{L}$, van Oers HA, Maurice-Stam $\mathrm{H}$, et al. Health related quality of life and parental perceptions of child vulnerability among parents of a child 
with juvenile idiopathic arthritis: results from a web-based survey. Pediatr Rheumatol Online J. 2014;12:34.

19. Van Roy B, Groholt B, Heyerdahl S, Clench-Aas J. Understanding discrepancies in parent-child reporting of emotional and behavioural problems: Effects of relational and socio-demographic factors. BMC Psychiatry. 2010;10:56.

20. Ringold S, Wallace CA, Rivara FP. Health-related quality of life, physical function, fatigue, and disease activity in children with established polyarticular juvenile idiopathic arthritis. J Rheumatol. 2009;36(6):1330-6.

21. Rochette E, Duché $P$, Merlin E. Juvenile idiopathic arthritis and physical activity: possible inflammatory and immune modulation and tracks for interventions in young populations. Autoimmun Rev. 2015;14(8):726-34.

Submit your next manuscript to BioMed Central and we will help you at every step:

- We accept pre-submission inquiries

- Our selector tool helps you to find the most relevant journal

- We provide round the clock customer support

- Convenient online submission

- Thorough peer review

- Inclusion in PubMed and all major indexing services

- Maximum visibility for your research

Submit your manuscript at www.biomedcentral.com/submit
Biomed Central 